\title{
From SNARC to SQUARC: Universal Mental Quantity Line?
}

\author{
Atanas Kirjakovski ${ }^{1} \&$ Narisuke Utsuki ${ }^{1}$ \\ ${ }^{1}$ Graduate School of Intercultural Studies, Kobe University, Kobe, Japan \\ Correspondence: Atanas Kirjakovski, Graduate School of Intercultural Studies, Kobe University, 1-2-1 \\ Tsurukabuto, Nada-ku, Kobe, 657-8501, Japan. Tel: 81-908-483-9966. E-mail: kirjakovski@stu.kobe-u.ac.jp
}

Received: January 9, 2012

Accepted: March 5, 2012 Published: June 1, 2012

doi:10.5539/ijps.v4n2p217

URL: http://dx.doi.org/10.5539/ijps.v4n2p217

\begin{abstract}
Numbers are represented spatially. Evidence for this comes from the SNARC effect (Spatial-Numerical Association of Response Codes). Small numerals are accessed faster on the left and large ones on the right. The effect assumes a mental number line. However, in A Theory of Magnitude (ATOM), both numerical (discrete) and non-numerical (continuous) quantities share a common processing mechanism. Thus, the SNARC effect may be an instance of a general SQUARC effect (Spatial-Quantity Association of Response Codes). We devised two experiments to test this hypothesis. First, 85 Japanese students bisected a quantity of water. They showed bisection bias by overestimating the right cup and underestimating the left one. In the second experiment, small continuous magnitudes shifted covert visual attention leftward, with larger ones shifting it rightward. Our data suggest that SNARC may be part of SQUARC, and the mental number line may be in fact a mental quantity line.
\end{abstract}

Keywords: SNARC, SQUARC, bisection, attentional shift, mental quantity line

\section{Introduction}

The perceptual and cognitive systems of humans and animals possess remarkable abilities to estimate environmental magnitudes. Both systems are crucial for successful survival of active organisms that perform cognitive and behavioral tasks. Specifically, external stimulations create constant environmental flux in which different sizes, quantities, and magnitudes have to be estimated efficiently. In the cognitive domain, humans have developed a highly organized and meaningful system of numerals, mostly used to express different discrete magnitudes. These symbols enable practical and conceptual calculations as well as estimations of varieties of environmental magnitudes to an enormous extent. For instance, we are able to estimate the sizes of elementary particles in atoms or the vast distances between celestial bodies. The numbers are precise in symbolizing magnitudes, even enabling us to understand concepts like infinity (e.g., the number " $\pi$ ").

Much research has been done on numerical cognition. One of the most influential findings in the field is the SNARC effect (Spatial-Numerical Association of Response Codes) described by Dehaene, Bossini, and Giraux (1993). Participants in that study judged the odd-even parity of Arabic numerals 0 to 9, and although irrelevant to the task, the numerical magnitude systematically influenced response times. Smaller numerals were responded to faster with response codes on the left, in contrast to larger numerals responded to faster with response codes on the right (for reviews see Gevers \& Lammertyn, 2005; Wood, Willmes, Nuerk, \& Fischer, 2008). This effect implies a mental number line (MNL; Moyer, 1973; Moyer \& Landauer, 1967; Restle, 1970) in which the numbers are represented on a spatial continuum with small magnitudes towards the left side and large magnitudes towards the right.

In addition to the SNARC effect, several similar effects have been reported in the literature. Most of them show spatially coded magnitudes. For instance, in the SMARC effect (Spatial-Musical Association of Response Codes), responses with the left key are faster for low-pitch sounds, while right key responses are faster for high-pitch sounds (Lidji, Kolinsky, Lochy, \& Morais, 2007; Nishimura \& Yokosawa, 2009; Rusconi, Kwan, Giordano, Umiltà, \& Butterworth, 2006). The left-to-right ascending spatial coding of magnitude extends to the temporal domain, as shown in the STEARC effect (Spatial-Temporal Association of Response Codes; Ishihara, Keller, Rossetti, \& Prinz, 2008). This effect was observed when Ishihara and colleagues instructed their participants to respond with horizontally aligned buttons to eight sequential auditory clicks, each fixed to $500 \mathrm{~ms}$. However, the last probe click was different from the previous seven and varied between early and late onset $( \pm 215 \mathrm{~ms})$. The task was to judge the onset of the eighth audio click. The early onset times elicited faster responses with the left key, with the late onsets eliciting faster responses with the right key. 
Behavioral data from the three effects indicate shared, spatially coded mental representations of magnitude in the numerical, auditory, and temporal domains. This similarity intuitively suggests that mental magnitude representation could be generalized. In other words, there may be a broader representational system of magnitude that processes any quantitative information equally, regardless of the modality. Gallistel and Gelman (2000) provided evidence from animal subjects for shared mental magnitudes:

Countable and uncountable quantity (numerosity and amount, duration, etc.) should be represented with the same kind of symbols (mental magnitudes), because there are many cases in which the two kinds of quantity must be combined arithmetically to determine behaviorally important decision variables. (Gallistel \& Gelman, 2000, p. 62)

This notion motivated Walsh (2003) to review a long list of studies from disciplines including cognitive psychology, neuropsychology, cognitive neuroscience, brain-imaging, developmental psychology, and research on animal subjects, and to summarize the findings in a single unifying theory named $A$ Theory of Magnitude (ATOM). ATOM hypothesizes the common processing mechanism of temporal, spatial, and numerical magnitudes located in the parietal cortex. Behavioral action is the link that connects the three magnitude types (Rossetti et al., 2004; Walsh, 2003).

Furthermore, considering countable and uncountable quantities as part of a common magnitude system, Walsh predicted that the SNARC effect could be an instance of a general quantity effect-SQUARC effect (Spatial-Quantity Association of Response Codes). That is, both discrete and continuous magnitudes share the same or similar mental representations.

To test this hypothesis, we performed two experiments that differed in their methodology. The first involved bisection of water quantity into two empty cups positioned left and right from the midline of the participant's body. The second experiment looked for differences in response times to visual cues with low and high magnitudes in a simple detection task. The null hypothesis of both experiments assumed absence of any organized mental representation of quantity. Alternatively, if the null hypothesis is false, then the behavioral data should show a pattern in which smaller magnitudes are associated with the left space, and larger magnitudes with the right space.

\section{Experiment 1: Bias in the Bisection of Water Quantity}

The SNARC effect assumes mental representation of numbers on a spatial continuum or mental number line (Dehaene, 1992). The smaller numerals are represented towards the left side of the number line, with the larger ones towards the right side (Dehaene, et al., 1993). Additionally, the spatial component of SNARC can influence spatial perceptions and actions in a variety of domains. For instance, it has been shown that it dramatically biases the allocation of covert visual attention (Fischer, Castel, Dodd, \& Pratt, 2003), shifting it towards the left for smaller numerals and towards the right for larger numerals. In addition, neurologically healthy participants show leftward pseudoneglect in line bisection tasks (Jewell \& McCourt, 2000). Nonetheless, if participants are asked to bisect strings made of the French words for "TWO (DEUX)" and "NINE (NEUF)", the leftward pseudoneglect increases in the case of TWO and significantly decreases in the case of NINE (Calabria \& Rossetti, 2005), showing automatic numerical magnitude activation.

If there is a common format for the mental representation of magnitude, we might expect mental transformations across quantitative dimensions. For example, brightness can be expressed in terms of handgrip pressure, line length, number, or loudness (Cantlon, Platt, \& Brannon, 2009). In addition, numeric values can influence temporal perception in that duration judgments paired with larger numeric magnitudes are reported to last longer (Xuan, Zhang, He, \& Chen, 2007). Indeed, neuroimaging studies show a neural substrate overlap for size, luminance, and number stimuli in comparative judgment tasks (Pinel, Piazza, Le Bihan, \& Dehaene, 2004).

The aim of this part of the study was to gather evidence for a generalized magnitude system (possible SQUARC effect). Based on this view, we designed a simple experiment in which we used water as a testable continuous quantity. In the context of the proposed SQUARC effect (Walsh, 2003), we suspect that not only discrete numerical, but all other non-numerical continuous quantities may be represented in an ascending left-to-right mental quantity line. Thus, our main hypothesis stated that the small magnitudes are associated with the left space and the large magnitudes with the space on the right. In addition, some studies have reported differences in performance between males and females on various tasks related to space, with males often showing better spatial abilities (Moffat, Hampson, \& Hatzipantelis, 1998; Voyer, Voyer, \& Bryden, 1995). Considering the spatial nature of the SNARC effect and other similar effects, it is also possible to hypothesize regarding sex differences in the current study. 


\subsection{Method}

\subsubsection{Participants}

A large number of students in the cafeteria of the Graduate School of Intercultural Studies at Kobe University agreed to participate $(N=85)$. Forty-five were females with a mean age of $19.73(S D=2.24)$, and 40 were males with a mean age of $20.23(S D=3.25)$. All participants showed preference for the right hand and declared themselves right-handed. Most of the students were Japanese, with a few others from different nationalities, not exceeding more than $10 \%$ of the sample. All participants provided written consent.

\subsubsection{Materials}

The materials for the experiment included six paper cups with a volume of $205 \mathrm{ml}$ each, Tanita digital scale model KD-400, a white paper strip $21 \mathrm{~cm}$ in length and $3 \mathrm{~cm}$ in width, and $150 \mathrm{ml}$ of tap water. Because a digital scale was used to measure the weight of the cups, and the density of tap water is very close to $1\left(0.99823 \mathrm{~g} / \mathrm{cm}^{3}\right.$ on $20^{\circ} \mathrm{C}$ ), weight expressed in grams would lead to a virtually equal number (in the case of this experiment 150 $\mathrm{ml} \approx 150 \mathrm{~g}$ ). Thus, the amount of water in all further analysis of the data will be expressed in grams instead of milliliters for the purpose of simplicity. Although six cups were mentioned, we reduced to three by inserting one into another to make the walls of the paper cups thicker to avoid any possibility of see through. The cup on the left side of the visual field of the participant was marked "L," and the cup on the right side was marked "R", written on the outer layer of the cup. The third cup had no marks on it. The cups were white and had no markings inside that participants could use as a reference point.

\subsubsection{Procedure}

For all participants, data were collected in the faculty cafeteria on a table under good lightening conditions and visibility. Participants who agreed to take part in the experiment were asked to sit at the table opposite the experimenter. The materials were already arranged on the table. The cup with the letter "L" was positioned on the left side of the participant's body midline. The cup with the letter "R" was on the participant's right, and the paper strip was placed in between the cups in a horizontal manner (Figure 1, A). The third unmarked cup containing $150 \mathrm{~g}$ of water was positioned in the center between the other two cups and approximately $10 \mathrm{~cm}$ from the horizontal strip. The left and right cups were empty. Participants were asked to sit comfortably, and their sagittal plane was approximated to the midpoint between the two cups. After acquiring information about their age and handedness, participants were given instructions on the task. They were asked to bisect the amount of water evenly from the third unmarked cup, between the left and right cup. Participants were allowed to choose which cup they would pour first, but were not allowed to place any of the cups next to each other. Each of the participants performed two trials (one for the left and one for the right hand). They were allowed to use only one hand at a time for each trial, and if they accidentally touched the cups with the unassigned hand for the trial, the procedure stopped, and they were asked to repeat the task from the beginning. Forty-three participants (23 females, 20 males) were instructed to use the right hand first, and 42 (22 females, 20 males) started with the left. The total number of trials for all participants was 170. After pouring the water into the left and right cups, the third cup was returned to its original position. When the initial relocation of the water into the other two cups was done, participants were allowed to adjust the amounts in the left and right cups to what they considered equal. They were not limited in time or adjustment moves. After they said they were finished, the weights of the left and right cup for each trial were measured and recorded, but were not shown to the participants until the end of the task in order not to influence their next performance. The whole procedure lasted approximately 10 minutes per participant.

\subsubsection{Data Analysis}

The analysis was performed on the right cup only, given that the scores obtained from both cups were interdependent. For example, if the measurement was $76 \mathrm{~g}$ in one cup, the other cup was automatically set to 74 g. Therefore, only the observations from the right cup were selected for statistical analysis. All conclusions hereafter resulted from the right cup. Each individual trial measurement was subtracted by $75(X i-75)$, and the obtained mean deviation was compared with the hypothetical population with mean equal to zero $(\mu=0)$. Possible effects of the following independent variables were considered: sex (males, females); hand (left, right); hand sequence (left hand first, right hand second; right hand first, left hand second); and any interaction (sex, hand, and hand sequence).

\subsection{Results}

For immediate recognition, the average weight was $74.3 \mathrm{~g}$ for the left and $75.6 \mathrm{~g}$ for the right cup (Figure 1, B). An ANOVA with repeated measures for hand (Sex $\times$ Hand Sequence $\times$ Hand [left, right]) was carried out on the 
dependent variable mean deviation from the hypothetical mean of $75(X i-75)$. The intercept reached significance, $F(1,81)=8.26, p=.005$. A significant effect was found for hand, $F(1,81)=5.59, p=.021$ (Figure $1, C)$. The main effects of sex and hand sequence approached significance: sex, $F(1,81)=2.92, p=.091$ and hand sequence, $F(1,81)=3.36, p=.071$. The interactions between Sex $\times$ Hand, Hand $\times$ Hand Sequence, and Sex $\times$ Hand $\times$ Hand Sequence were not significant.

Although the ANOVA for the independent variable of sex did not reach significance at the specified alpha .05 level, additional one sample t-tests showed that the scores obtained from males (with the right hand) were significantly different from the hypothetical mean of zero $(\mu=0), t(39)=3.61, p<.001$. However, this was not evident in the right hand scores for females, $t(44)=1.51, p=.137$ (Figure 1, D). In the case of hand sequence, the one sample t-test showed that when the right hand was used first, the scores for right hand were significantly different from zero, $t(42)=3.03, p=.004$. All other combinations were not statistically significant.

\subsection{Discussion}

In general, a strong unilateral bias for the right hand was especially apparent among male participants (left cup was light-weighted in relation to the right cup), but not among females. The hypothesis was tested using water as a continuous quantity to be allocated evenly between two cups, that is, to be bisected. The task is analogous to the well established bisection task often used in neuropsychology and neuroscience (Fischer, 2001). Furthermore, Jewell and McCourt (2000) conducted an extensive meta-analysis of 73 studies (or sub-studies) involving 2,191 subjects in which variations of a bisection task were explored, including visual and non-visual line bisection, midsagittal-pointing tasks, and tactile bisection tasks. They concluded that all lead to leftward bisection errors (i.e., leftward pseudoneglect). In addition, the researchers inferred that bisection errors are weakly affected by the handedness of the subjects with dextrals erring slightly further to the left than sinistral subjects, and male subjects making slightly larger leftward errors than female subjects in some studies (e.g., Roig \& Cicero, 1994). The same mechanisms that cause pseudoneglect could potentially offer an explanation for the findings in the current study.

In relation to the task used in this study, several important points are worth mentioning. Different participants used different strategies to estimate the quantities in the cups. Some inclined their bodies towards the cup of interest or weighted the cups interchangeably with the allowed hand for the trial. Some participants poured water incrementally, and some allocated the total amount of the water into one of the cups and then relocated small decrements into the other cup. All strategies did have something in common, especially for males and their right hand, which conversely implies an underlying mechanism powerful enough to overcome the variation in individual strategies. Consequently, it can be concluded that the estimated water quantity followed the SNARC pattern, advocating the possible existence of the SQUARC effect as a supracategory of other numerical, spatial, quantity, and magnitude-related association codes. In other words, ideas about common cortical metrics of time, space, and quantity (Walsh, 2003) rely on a common representational format for different magnitudes (Cantlon et al., 2009) as well as plasticity and interchangeability between the formats.

However, there are a few points that we should criticize. Cups used in the experiment were marked "L" for the left cup and " $\mathrm{R}$ " for the right cup. It is unclear if the side (left or right) had a cuing effect on estimation of water quantity, with the concept of "left" being associated with small magnitudes and "right" with large ones. In addition, the sequence of allocating water between the cups, as a function of the choice of the participant, might be of great importance. In this experiment, it might have provided information about the subjective referential point for the estimation, with all subsequent choices and comparisons possible being executed in relation to this point. For example, if a participant started to pour water in the left cup first, this could imply a subjective "zero-point" as a spatial landmark for the small magnitudes. Unfortunately, data for the choice of the first cup were not collected.

In summary, participants in this experiment did not bisect the water from the third cup into the left and right cup evenly. On the contrary, they overestimated the amount of water in the right cup. It seems that not only numerals (discrete information) exhibit bisection bias. Other types of continuous quantities obey the same rules and generalize into a possible SQUARC effect.

\section{Experiment 2: Spatial Shifts of Covert Visual Attention Cued by Non-numerical, Continuous Magnitudes}

In the water bisection experiment, participants overestimated the water quantity in the right cup and underestimated the left one. This finding suggests that non-numerical continuous quantities may be mentally represented in the form of a mental quantity line with small quantities on the left and large quantities on the right. 
Although the first experiment provided general insight into the cognitive representation of non-numerical, continuous magnitudes, in the far more studied numerical domain, numerical representation often affects performance on numerous behavioral tasks. For example, the semantic size of the numeral can interfere with its physical size, and vice versa (Henik \& Tzelgov, 1982). In addition, numerical magnitude appears to affect the choice of the first gaze direction when participants are allowed to scan the screen freely after the initial presentation of numerals 1 to 9 (Fernández, Rahona, Hervás, Vázquez, \& Ulrich, 2010).

Mere exposure to numerals can influence human perception. One remarkable finding is the shift of covert visual attention to the left or right merely by presenting numerals with different magnitudes (Fischer et al., 2003). Fischer et al. took advantage of the spatial cuing paradigm (Posner, 1980; Posner, Snyder, \& Davidson, 1980) and demonstrated that participants are faster to respond to the left target when cued by centrally presented small numerals $(1,2)$, and respond faster to the right target when cued by larger numerals $(8,9)$. It appears that in the findings of Fischer et al., numerals serve as relevant cues for location, creating certain expectancy in the observer, revealed through the congruence in faster response times for the small magnitudes and the left target, and incongruence for the target on the right.

Thus, some specific dimension of the numerals must be activating top-down, endogenous control of attention. This control relies on the already mentioned mental number line (MNL). The MNL is externalized and elongated into the extracorporeal space, creating a spatial expectancy map. Consequently, associating large numerals with the left side of the space creates an unexpected event that generates some cost in response times.

Additionally, Ito and Hatta (2004) documented the existence of the SNARC effect among 30 Japanese students who participated in their study, providing an argument for the cultural robustness of the effect. Consequently, we made an effort to replicate the findings of Fischer et al. (2003) with Japanese participants. However, Fischer et al. instructed the participants to use their preferred hand throughout the experiment. In our case, response times were measured for both hands independently. This modification was based on the suspicion of Mapelli, Rusconi, and Umiltà (2003) who related the Simon effect (Simon, 1969) to the SNARC effect. In their experiment, Mapelli et al. presented the numbers from 1 to 9 (without 5) laterally, left and right from the fixation point in a parity judgment task. They found that when the numbers were presented on the left side, response times executed with the left hand were faster than the ones with the right, and vice versa.

In this experiment, we aimed to replicate the findings of Fischer et al. (2003) in which the right target was detected faster when preceded by a large numeral, opposite to when preceded by a small numeral. Furthermore, we tested the prediction of Walsh (2003) for possible existence of a broader category of response codes (i.e., SQUARC effect). In the main hypothesis we reasoned that if the magnitude of numbers denotes the SNARC effect, under the presumption of a more generalized SQUARC effect, the same effect will occur with a non-numerical, continuous type of stimuli.

\subsection{Method}

\subsubsection{Participants}

Twenty native Japanese students $(N=20)$ at the Graduate School of Intercultural Studies, Kobe University, responded to the written request and agreed to participate in this study. The sex ratio was balanced to 10 males and 10 females, with a mean age of $19.9(S D=0.99)$ for males and $20.1(S D=1.79)$ for females. The Edinburgh Inventory (Oldfield, 1971) was used to assess the handedness of the participants. Results showed a laterality quotient (LQ) of +91 on average (males $=+90.5$; females $=+91.5$ ). This classified all participants as right-handed. All participants reported normal or corrected-to-normal vision. The testing time for each individual participant lasted approximately $30 \mathrm{~min}$.

\subsubsection{Apparatus and Stimuli}

The equipment for this experiment consisted of one computer, 17" LCD monitor (5:4 aspect ratio; resolution $1280 \times 1024)$, chin rest, and chair with adjustable height. For the presentation of stimuli and measurement of response time (RT), we used SuperLab Pro (Version 2.0.4) for Windows and a JIS standard layout PC keyboard. The stimuli set contained Arabic numerals 1, 2, 8, and 9 (size $1.5^{\circ}$ ); graphical image of an empty and full wine glass (width $0.8^{\circ}$ and height $1.8^{\circ}$ ); and short and long horizontal line (width $3^{\circ}, 9^{\circ}$; height $0.3^{\circ}, 0.3^{\circ}$, respectively) (Figure 2, A). Each trial started with a central fixation point $\left(0.2^{\circ}\right.$ in diameter) placed between 2 white squares (each had $10^{\circ}$ eccentricity from the fixation point and $2^{\circ}$ width). Finally, a target (white circle, $1.4^{\circ}$ in diameter) appeared in one of the squares. All stimuli were presented on a black background.

\subsubsection{Procedure}

After participants were given instructions and completed The Edinburgh Inventory, the experimenter led them to 
another room where the experiment took place. They were told to take a seat and place their chin on the chin rest fixed at $50 \mathrm{~cm}$ from the LCD monitor. Next, they were asked to adjust the height of the chair to a comfortable level. Participants were instructed to maintain their gaze towards the center of the screen and were encouraged to respond with a central button-press as fast as possible after target detection. With their preferred hand, the participants practiced 24 trials. The experimental session consisted of 56 randomized experimental trials with the left hand first, and another 56 trials with the right, totaling 2,240 trials for all participants. The total number of trials included 14\% catch trials (where no target appeared) and $86 \%$ valid trials (target appeared). The catch trial error rate was very low $(0.14 \%)$.

All participants were informed that none of the stimuli predicted the location of the target, and were asked to make a button-press as quickly and accurately as possible each time a target appeared in one of the two squares. Each trial began with a centered fixation point $(500 \mathrm{~ms})$ that was replaced randomly with one of the four numbers, wine glass, or horizontal line $(300 \mathrm{~ms})$. Then, the same fixation point reappeared for another $450 \mathrm{~ms}$. Finally, a white target circle was presented randomly in the left or right square (Figure 2, B). It is important to note that in the original experiment, Fischer et al. (2003) used a random delay of 50, 100, 200, 300, 400, and 500 $\mathrm{ms}$ between offset of the stimulus and onset of the target. However, they reported significant effects only for delays of 400 and $500 \mathrm{~ms}$. Thus, in our experiment, the delay was fixed to $450 \mathrm{~ms}$ between the stimulus and target.

\subsubsection{Data Analysis}

The response times between $200 \mathrm{~ms}$ and three standard deviations above the mean were taken into consideration (calculated for each type of stimuli, separately for both hands). Because the wine glass and horizontal line appeared 4 times randomly within each condition (e.g., empty wine glass appeared 4 times; full wine glass 4 times), we calculated mean RT for each and summarized the calculations into a data set of the response times, excluding the outliers and errors.

Accordingly, the numerical stimuli were classified as "low" $(1,2)$ and "high" $(8,9)$ magnitudes. Additionally, the pairs of "incongruent" and "congruent" conditions were compared (e.g., comparison of the response times for the conditions "empty wine glass - target left, with "full wine glass - target left" since the only dimension that varied was the magnitude of the wine in the glass).

\subsection{Results}

Three separate $2 \times 2 \times 2$ (Hand [left, right] $\times$ Target Location [left, right] $\times$ Congruency [incongruent, congruent]) repeated-measures ANOVAs were performed for each kind of stimuli. In the case of the numerals, a significant main effect was found for hand, $F(1,19)=4.79, p=.041$, coupled with a Hand $\times$ Target Location interaction $F(1$, $19)=9.31, p=.007$. The main effect of congruency was evident for the wine glass stimuli $F(1,19)=8.11, p$ $=.010$. The Target Location $\times$ Congruency interaction for the horizontal line reached significance, $F(1,19)=$ $6.76, p=.018$ (Figure 2, C). No other main effects or interactions were significant.

\subsection{Discussion}

One of the purposes of this experiment was to replicate the findings of Fischer et al. (2003). In our case, the main effect of congruency was not significant in the numerals condition. Thus, there was not sufficient evidence to replicate that study's findings. Although the SNARC effect was not evident in the numerals condition, in general, response times executed with the right hand were faster than ones executed with the left. This might be attributed to the right-handedness of the participants or simply an effect of learning throughout the experimental session. Additionally, the significant Hand $\times$ Target Location interaction revealed the Simon effect, (i.e., RTs for the left hand were shorter for the target on the left, which was also true for the right hand and target on the right). As a result, when the spatial cuing paradigm was coupled with the simple detection task in the study of the numbers, there might have been an underlying bias in the form of the Simon effect, which on the other hand, might have obscured the real existence of the SNARC effect. For instance, RT differences for the targets presented left or right from the center in the numerals condition may have been due to the faster responses of the left hand for the left targets and right hand for the right targets, (i.e., the Simon effect). Therefore, it is not clear whether the cuing power of the numerals shifted the spatial orientation of covert attention (i.e., SNARC effect). The other two types of stimuli (wine glass and horizontal line) did not show significant Hand $\times$ Target Location interaction. Therefore, there is insufficient evidence to speculate about a possible Simon effect.

Contrary to the findings for numerical stimuli, the wine glass showed a clear main effect of congruency. In other words, when the empty wine glass preceded a target on the left side, participants were faster to respond. By the same token, when the full wine glass came before the target, the RTs for the right target were faster. It is clear 
that the wine glass acted as a valid cue for the spatial shifts of covert attention. In other words, we can conclude that the prediction of Walsh (2003) regarding the existence of a broader category of response codes for spatial associations (i.e., SQUARC) was supported. Consequently, the continuous magnitude of the wine glass could give some insights into the mental representation for this type of quantity, and could help psychologists understand that a mental number line (Moyer, 1973; Moyer \& Landauer, 1967; Restle, 1970) is actually a mental quantity line, which is not solid but rather has a fluid-like nature.

Similarly, but with slightly different magnitude quality, the horizontal line had a different outcome than the SQUARC effect would predict. The only statistically significant component was the Target Location $\times$ Congruency interaction. When the horizontal line was used as a cue, for the target presented on the left side, the congruent cue (short line) caused slower response times. That is, a reversed SQUARC effect was observed. At this point, we cannot offer a satisfactory explanation for this observation. However, while the magnitude of the wine glass is contained within the volume of the container, the horizontal line is perceived as a one-dimensional object, and therefore, has only magnitude of length. Speculatively, when consecutively presented, the short and long lines may have seemingly overlapped, and it may have looked as if they converged towards the vertex of the screen. Creating a depth illusion, they may have actually served as cues for depth instead of cues for length. Consequently, participants may have perceived the two lines as farther apart from each other. Because the short line looked more distant, what was actually perceived was the depth magnitude, not the length magnitude of the line per se. If this interpretation is true, it is in agreement with the SQUARC hypothesis. In addition, the horizontal line varied in length and occupied different positions on the screen. It shrank and expanded, perhaps inducing different attentional processing in comparison to the centrally fixed numerals and wine glass.

Even though horizontal line and numerical stimuli did not yield the hypothesized results, the wine glass stimuli showed straightforward spatial coding of continuous magnitude. Taken all together, it can be concluded that presentation of non-numerical, continuous stimuli in the form of a wine glass with voluminous magnitude can influence the spatial shifts of covert attention. We believe that this finding is one step closer towards clarification of the issue of whether the SNARC effect is really an instance of a more generalized SQUARC effect.

\section{Conclusion}

At this point, it is not quite clear whether the mental representation of numerals transfers to the mental representation of general quantity (i.e., SNARC transfers to SQUARC). However, in Experiments 1 and 2, we provide evidence that non-numerical, continuous quantities are not randomly or symmetrically distributed, but there is certain cognitive organization. Similar to numerals, other types of quantity seem to be spatially represented with small magnitudes placed towards the left space, and large magnitudes towards the right.

Still, there is a long road ahead in the discovery of how the brain represents quantity. This is an arduous task and necessitates strong evidence from different disciplines. Nevertheless, the SNARC effect has established itself as a firm guidepost for where to look for answers. It seems that numerical magnitude is organized in ascending left-to-right fashion (i.e., mental number line). Educated adults may acquire this mental representation through the repeated use of numbers, enforced by overlearned reading and writing.

Even so, before the mental number line becomes a mental quantity line, one question remains: How does this mental organization aid the brain in quantity processing, and what is its significance for behavior? Future studies may shed light on answers to these questions. 
A.

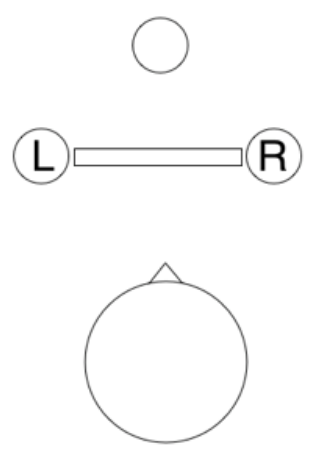

B.

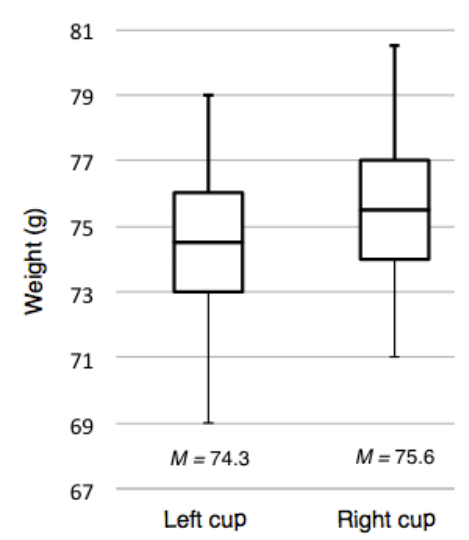

C.

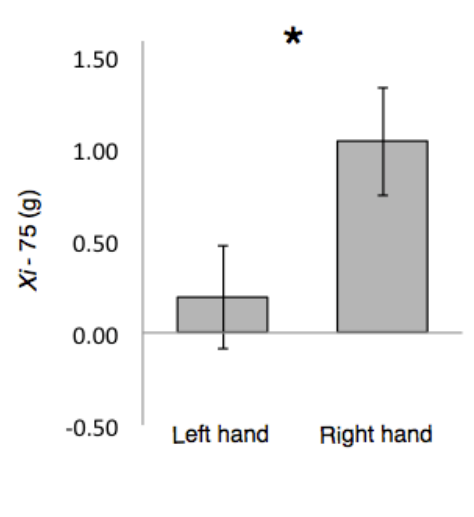

D.

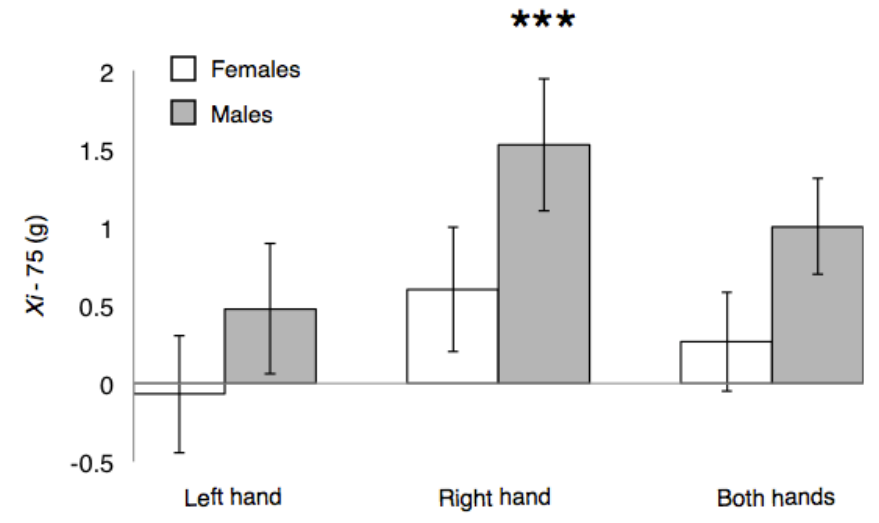

Figure 1. Water Quantity Bisection Task

A. Position of the cups in relation to participant. B. The average weight of the left and right cup. Participants overestimated the right cup and underestimated the left cup (the midpoint was equal to $75 \mathrm{~g}$ ). C. Main effect of the hand. When participants used the right hand, they were more prone to overestimate the right cup and underestimate the left one. The analysis was performed on individual score deviations from the hypothetical midpoint of $75(X i-75)$. D. Although the ANOVA analysis did not reveal significant results at an alpha level of .05 for either sex, an additional one sample t-test confirmed bisection bias in males, contrary to females. The bias was evident as overestimation of the right cup, particularly when participants used their right hand. The error bars represent standard errors of the mean.

$* p<.05 . * * * p<.001$. 
A.

\begin{tabular}{ccc}
\hline$\square$ & 2 & $\square$ \\
\hline$\square$ & 9 & $\square$ \\
\hline$\square$ & 9 & $\square$ \\
\hline$\square$ & 1 & $\square$ \\
\hline$\square$ & - & $\square$ \\
\hline$\square$ & - & $\square$ \\
\hline
\end{tabular}

B.

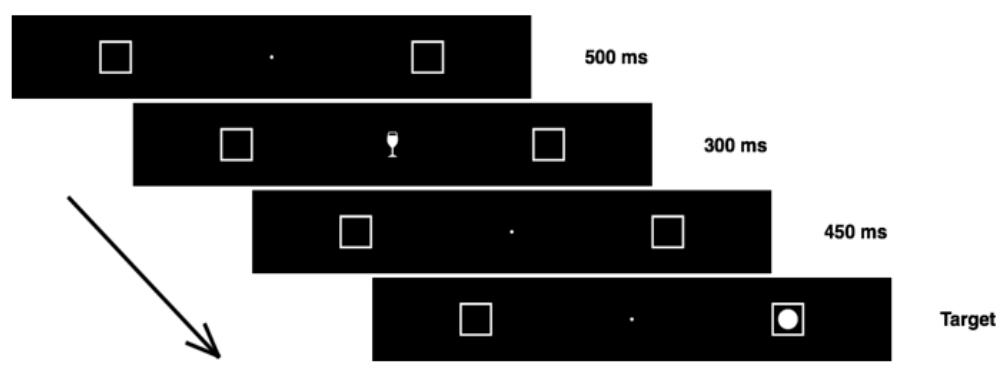

c.

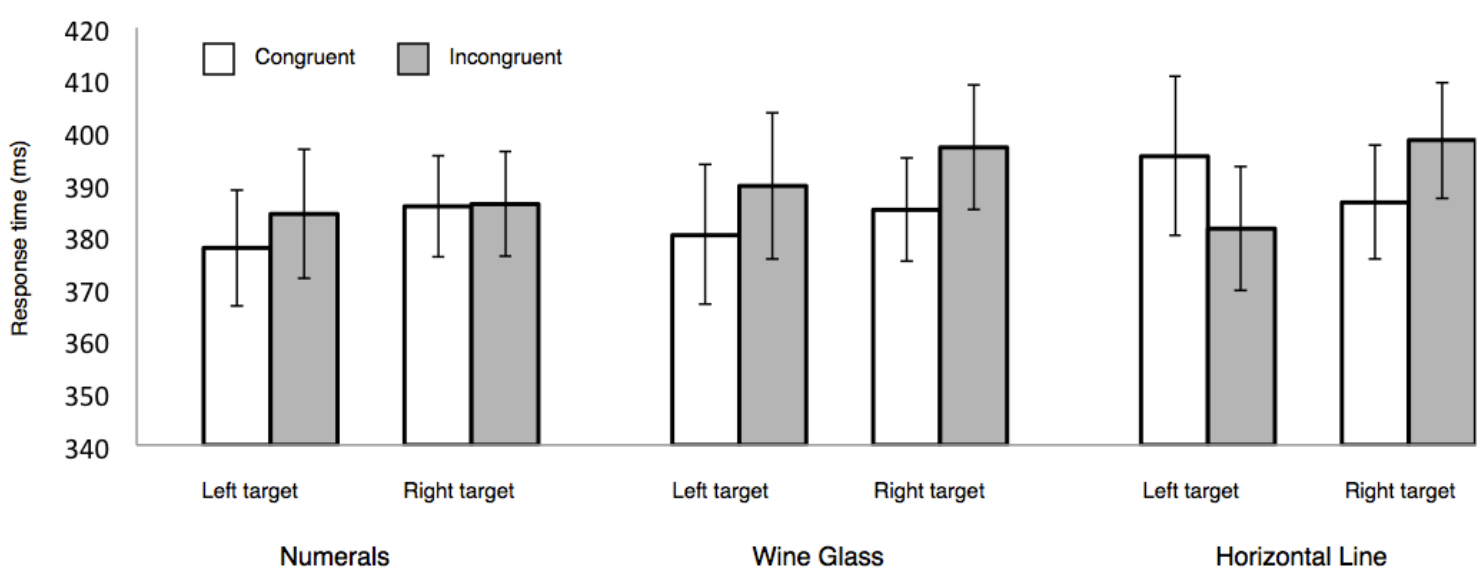

Figure 2. Spatial Shifts of Covert Visual Attention

A. Example of the stimuli used in Experiment 2. The total set of stimuli contained the numerals 1, 2, 8, and 9, empty/full wine glasses, and short/long horizontal lines. B. Each trial began with a centered fixation point (500 $\mathrm{ms}$ ) that was replaced randomly with one of the four numbers, wine glass, or horizontal line (300 ms). Then the same fixation point reappeared for another $450 \mathrm{~ms}$. Finally, the target was presented randomly in the left or right square. C. In the numerals condition, the SNARC effect was evident only for the left target. We partially succeeded in replicating the findings of Fischer et al. (2003). The main effect of congruency was evident for the wine glass stimuli. The response times were faster when the magnitude (empty/full wine glass) and target location (left/right) were congruent. In other words, when an empty wine glass came before the target on the left, participants were faster to respond. The same was true for a full wine glass and target on the right. In the horizontal line condition, although statistically significant, results were mixed. The error bars represent standard errors of the mean.

\section{References}

Calabria, M., \& Rossetti, Y. (2005). Interference between number processing and line bisection: A methodology. Neuropsychologia, 43(5), 779-783. http://dx.doi.org/10.1016/j.neuropsychologia.2004.06.027

Cantlon, J. F., Platt, M. L., \& Brannon, E. M. (2009). Beyond the number domain. Trends in Cognitive Sciences, 13(2), 83-91. http://dx.doi.org/10.1016/j.tics.2008.11.007

Dehaene, S. (1992). Varieties of numerical abilities. Cognition, 44(1-2), 1-42. http://dx.doi.org/10.1016/0010-0277(92)90049-N

Dehaene, S., Bossini, S., \& Giraux, P. (1993). The mental representation of parity and number magnitude. Journal of Experimental Psychology: General, 122(3), $371-396$. http://dx.doi.org/10.1037/0096-3445.122.3.371 
Fernández, S. R., Rahona, J. J., Hervás, G., Vázquez, C., \& Ulrich, R. (2010). Number magnitude determines gaze direction: Spatial-numerical association in a free-choice task. Cortex, 47(5), 617-620. http://dx.doi.org/10.1016/j.cortex.2010.10.006

Fischer, M. H. (2001). Cognition in the bisection task. Trends in Cognitive Sciences, 5(11), 460-462. http://dx.doi.org/10.1016/S1364-6613(00)01790-3

Fischer, M. H., Castel, A. D., Dodd, M. D., \& Pratt, J. (2003). Perceiving numbers causes spatial shifts of attention. Nature Neuroscience, 6(6), 555-556. http://dx.doi.org/10.1038/nn1066

Gallistel, R. C., \& Gelman, R. (2000). Non-verbal numerical cognition: From reals to integers. Trends in Cognitive Sciences, 4(2), 59-65. http://dx.doi.org/10.1016/S1364-6613(99)01424-2

Gevers, W., \& Lammertyn, J. (2005). The hunt for SNARC. Psychology Science, 47, 10-21.

Henik, A., \& Tzelgov, J. (1982). Is three greater than five: The relation between physical and semantic size in comparison tasks. Memory \& Cognition, 10(4), 389-395. http://dx.doi.org/10.3758/BF03202431

Ishihara, M., Keller, P. E., Rossetti, Y., \& Prinz, W. (2008). Horizontal spatial representations of time: Evidence for the STEARC effect. Cortex, 44(4), 454-461. http://dx.doi.org/10.1016/j.cortex.2007.08.010

Ito, Y., \& Hatta, T. (2004). Spatial structure of quantitative representation of numbers: Evidence from the SNARC effect. Memory \& Cognition, 32(4), 662-673. http://dx.doi.org/10.3758/BF03195857

Jewell, G., \& McCourt, M. E. (2000). Pseudoneglect: A review and meta-analysis of performance factors in line bisection tasks. Neuropsychologia, 38(1), 93-110. http://dx.doi.org/10.1016/S0028-3932(99)00045-7

Lidji, P., Kolinsky, R., Lochy, A., \& Morais, J. (2007). Spatial associations for musical stimuli: A piano in the head? Journal of Experimental Psychology: Human Perception and Performance, 33(5), 1189-1207. http://dx.doi.org/10.1037/0096-1523.33.5.1189

Mapelli, D., Rusconi, E., \& Umiltà, C. (2003). The SNARC effect: An instance of the Simon effect? Cognition, 88(3), B1-B10. http://dx.doi.org/10.1016/S0010-0277(03)00042-8

Moffat, S. D., Hampson, E., \& Hatzipantelis, M. (1998). Navigation in a "virtual" maze: Sex differences and correlation with psychometric measures of spatial ability in humans. Evolution and Human Behavior, 19(2), 73-87. http://dx.doi.org/10.1016/S1090-5138(97)00104-9

Moyer, R. S. (1973). Comparing objects in memory: Evidence suggesting an internal psychophysics. Attention, Perception, \& Psychophysics, 13(2), 180-184. http://dx.doi.org/10.3758/BF03214124

Moyer, R. S., \& Landauer, T. K. (1967). Time required for judgements of numerical inequality. Nature, 215, 1519-1520. http://dx.doi.org/10.1038/2151519a0

Nishimura, A., \& Yokosawa, K. (2009). Effects of laterality and pitch height of an auditory accessory stimulus on horizontal response selection: The Simon effect and the SMARC effect. Psychonomic Bulletin \& Review, 16(4), 666-670. http://dx.doi.org/10.3758/PBR.16.4.666

Oldfield, R. C. (1971). The assessment and analysis of handedness: The Edinburgh inventory. Neuropsychologia, 9(1), 97-113. http://dx.doi.org/10.1016/0028-3932(71)90067-4

Pinel, P., Piazza, M., Le Bihan, D., \& Dehaene, S. (2004). Distributed and overlapping cerebral representations of number, size, and luminance during comparative judgments. Neuron, 41(6), 983-993. http://dx.doi.org/10.1016/S0896-6273(04)00107-2

Posner, M. I. (1980). Orienting of attention. Quarterly Journal of Experimental Psychology, 32(1), 3-25. http://dx.doi.org/10.1080/00335558008248231

Posner, M. I., Snyder, C. R., \& Davidson, B. J. (1980). Attention and the detection of signals. Journal of Experimental Psychology: General, 109(2), 160-174. http://dx.doi.org/10.1037/0096-3445.109.2.160

Restle, F. (1970). Speed of adding and comparing numbers. Journal of Experimental Psychology, 83(2), 274-278. http://dx.doi.org/10.1037/h0028573

Roig, M., \& Cicero, F. (1994). Hemisphericity style, sex, and performance on a line-bisection task: An exploratory study. Perceptual and Motor Skills, 78, 115-120. http://dx.doi.org/10.2466/pms.1994.78.1.115

Rossetti, Y, Jacquin-Courtois, S., Rode, G., Ota, H., Michel, C., \& Boisson, D. (2004). Does action make the link between number and space representation? Visuo-manual adaptation improves number bisection in 
$\begin{array}{llll}\text { unilateral } \quad \text { neglect. } & \text { Psychological } & \text { Science, }\end{array}$ http://dx.doi.org/10.1111/j.0956-7976.2004.00696.x

Rusconi, E., Kwan, B., Giordano, B. L., Umiltà, C., \& Butterworth, B. (2006). Spatial representation of pitch height: the SMARC effect. Cognition, 99(2), 113-129. http://dx.doi.org/10.1016/j.cognition.2005.01.004

Simon, R. J. (1969). Reactions toward the source of stimulation. Journal of Experimental Psychology, 81(1), 174-176. http://dx.doi.org/10.1037/h0027448

Voyer, D., Voyer, S., \& Bryden, M. P. (1995). Magnitude of sex differences in spatial abilities: A meta-analysis and consideration of critical variables. Psychological Bulletin, 117(2), 250-270. http://dx.doi.org/10.1037/0033-2909.117.2.250

Walsh, V. (2003). A Theory of Magnitude: Common cortical metrics of time, space and quantity. Trends in Cognitive Sciences, 7(11), 483-488. http://dx.doi.org/10.1016/j.tics.2003.09.002

Wood, G., Willmes, K., Nuerk, H.-C., \& Fischer, M. H. (2008). On the cognitive link between space and number: A meta-analysis of the SNARC effect. Psychology Science Quarterly, 50(4), 489-525.

Xuan, B., Zhang, D., He, S., \& Chen, X. (2007). Larger stimuli are judged to last longer. Journal of Vision, 7(10), 1-5. http://dx.doi.org/10.1167/7.10.2 